IFUM-643-FT

\title{
Three-graviton scattering and recoil effects in M-atrix theory
}

\author{
Andrea Refolli, Niccolo' Terzi and Daniela Zanon \\ Dipartimento di Fisica dell'Università di Milano and \\ INFN, Sezione di Milano, via Celoria 16, I-20133 Milano, Italy
}

\begin{abstract}
We study the scattering of three gravitons in $M$-atrix theory at finite $N$. With a specific choice of the background we obtain the complete result up to two loops. The contributions from three-body forces agree with the ones presented in recent papers. We extend the calculation and evaluate the two-body exchanges as well. Such terms, somewhat difficult to isolate and compute, had been neglected so far in the existing literature. We show that the result we have obtained from $M$-atrix theory precisely matches the result from one-particle reducible tree diagrams in eleven-dimensional supergravity .
\end{abstract}

e-mail: andrea.refolli@mi.infn.it

e-mail: terzi@pcteor1.mi.infn.it

e-mail: daniela.zanon@mi.infn.it

May 1999 


\section{Introduction}

Since the introduction of the matrix model of $M$-theory [1] much work and much progress have been made on the subject. According to the original conjecture, the degrees of freedom of $M$-theory in the infinite momentum frame are contained in the dynamics of $N D$-branes in the $N \rightarrow \infty$ limit. Subsequently it was argued that $M$-theory with one of the lightlike coordinate compactified, the so called discrete light cone sector, is in fact equivalent to the super Yang-Mills matrix model for finite $N$ [2], [3]. Compelling tests of this proposal have been the comparison of two-body [4, 5] and three-body [6, 7, 8, 9] scattering.

We focus on the $M$-atrix theory at finite $N$ and explore further its correspondence with eleven dimensional supergravity. In particular we consider $N=3$ and compute graphs up to two-loops in $(0+1)$-dimensional Yang-Mills. We find that they perfectly reproduce the three graviton scattering in supergravity, both in the direct three-body channel and in the two-body recoil exchange. The former, which corresponds to one-particle irreducible diagrams in supergravity, has been computed in the first paper of ref. [6] and we confirm that result. We complete the two-loop effective action calculation and evaluate two-body exchanges as well. We have found a systematic way to separate the light and heavy matrix model degrees of freedom which allows to obtain the full answer. We show that these contributions exactly match what expected from the two-loop scattering of two $D 0$-branes in $M$-atrix theory [5].

We note that the recoil effects we have computed are in the context of an effective action calculation, i.e. two-loop 1P-irreducible diagrams in Matrix theory. In the second paper of ref. [6] a detailed analysis of recoil interpreted as geodesic acceleration due to one-loop corrections was given. These contributions correspond in Matrix theory to two-loop 1P-reducible diagrams.

In the next section we derive the explicit form of the gauged fixed action. The various fields are decomposed in terms of components on a $U(3)$ basis of hermitean matrices. The classical background is fixed with the three $D$-particles having relative velocities parallel to each others, orthogonal to the corresponding relative displacements. The Feynman rules are easily derived. In section 3 we give the two-loop effective action, leaving most of the technical details in the Appendices. The result is analyzed keeping separate the two types of contributions mentioned above, i.e. terms which depend on two distinct relative velocities (three-body interaction) and terms in which only one relative velocity appears (recoil). The last section contains a summary of our results. We have performed part of the calculations with the help of Mathematica. 


\section{The action}

As anticipated in the introduction, the matrix model is simply obtained by reducing $(9+1)$ dimensional $U(N)$ super Yang-Mills [10] to $(0+1)$ dimensions. This theory describes a system of $N D 0$-branes [11] in terms of nine bosonic fields $X_{i}$ and of sixteen fermionic superpartners $\theta$, which are spinors under $S O(9)$. The euclidean action is given by

$$
\begin{array}{r}
S=\operatorname{Tr} \int d \tau\left\{\left(D_{\tau} X_{i}\right)^{2}-\frac{g}{2}\left[X_{k}, X_{j}\right]\left[X_{k}, X_{j}\right]\right. \\
\left.+\theta^{T} D_{\tau} \theta-\sqrt{g} \theta^{T} \gamma^{k}\left[X_{k}, \theta\right]\right\}
\end{array}
$$

where we have denoted by $g$ the Yang-Mills coupling constant and by $\gamma^{i}$ nine real, symmetric gamma matrices satisfying $\left\{\gamma^{i}, \gamma^{j}\right\}=2 \delta^{i j}$. The covariant derivative is defined by

$$
D_{\tau}=\partial_{\tau}-i \sqrt{g}[A, \quad]
$$

The fields $X_{i}, \theta$ and $A$ are $N \times N$ hermitean matrices of $U(N)$, with $i, j, k=1,2, \ldots, 9$.

Being interested in quantum, perturbative calculations it is convenient to use the background field method, which allows to maintain explicit the gauge invariance of the result. To this end one expands the action (2.1) around a classical background field configuration $B_{i}$, setting $X_{i} \rightarrow X_{i}+B_{i}$. After gauge-fixing and trivial rescaling of the fields, the complete action is

$$
\begin{aligned}
S=\operatorname{Tr} & \int d \tau\left\{\left(\partial_{\tau} X_{i}\right)^{2}-\left[B_{k}, X_{j}\right]^{2}-2 \sqrt{g}\left[B_{k}, X_{j}\right]\left[X_{k}, X_{j}\right]-\frac{g}{2}\left[X_{k}, X_{j}\right]\left[X_{k}, X_{j}\right]\right. \\
& +\partial_{\tau} A^{2}-\left[A, B_{k}\right]^{2}-4 i \partial_{\tau} B_{k}\left[A, X_{k}\right]-2 i \sqrt{g} \partial_{\tau} X_{k}\left[A, X_{k}\right]+2 \sqrt{g}\left[A, B_{k}\right]\left[X_{k}, A\right] \\
& -g\left[A, X_{k}\right]^{2}+\theta^{T} \partial_{\tau} \theta-i \sqrt{g} \theta^{T}[A, \theta]-\sqrt{g} \theta^{T} \gamma^{k}\left[X_{k}, \theta\right]-\theta^{T} \gamma^{k}\left[B_{k}, \theta\right] \\
& \left.-2 \tilde{G} \partial_{\tau}^{2} G-2 i \sqrt{g} \partial_{\tau} \tilde{G}[A, G]+2 \tilde{G}\left[B_{k},\left[B_{k}, G\right]-\sqrt{g}\left[G, X_{k}\right]\right]\right\}
\end{aligned}
$$

Here $X_{i}, A$ and $\theta$ are the quantum fluctuations, $G$ and $\tilde{G}$ are the ghosts, while $B_{k}$ is the external background.

Since we want to extract results to be compared to the scattering of three gravitons in supergravity, the minimal choice for the Yang-Mills gauge group that allows to describe the interaction of three $D 0$-branes, is $U(3)$. In Appendix A we explicitly give the Cartan basis $H^{1}, H^{2}, E_{\alpha}$ (where $\alpha= \pm \alpha^{1}, \pm \alpha^{2}, \pm \alpha^{3}$ are the roots), and the commutator algebra.

Using such a basis every matrix field is decomposed into components as

$$
X_{k} \equiv X_{k}^{a} H_{a}+X_{k}^{\alpha} E_{\alpha}+X_{k}^{* \alpha} E_{-\alpha}
$$




$$
\begin{aligned}
A & \equiv A^{a} H_{a}+A^{\alpha} E_{\alpha}+A^{* \alpha} E_{-\alpha} \\
\theta & \equiv \theta^{a} H_{a}+\theta^{\alpha} E_{\alpha}+\theta^{* \alpha} E_{-\alpha} \\
G & \equiv G^{a} H_{a}+G^{\alpha} E_{\alpha}+G^{* \alpha} E_{-\alpha} \\
\tilde{G} & \equiv \tilde{G}^{a} H_{a}+\tilde{G}^{* \alpha} E_{\alpha}+\tilde{G}^{\alpha} E_{-\alpha}
\end{aligned}
$$

In order to keep the notation simple we use a greek index $\alpha=1,2,3$ as a suffix to enumerate some of the component fields and the same index to indicate the corresponding root on the matrices, i.e. $E_{1} \equiv E_{\alpha^{1}}, E_{2} \equiv E_{\alpha^{2}}, E_{3} \equiv E_{\alpha^{3}}$. Similarly, in the following of the paper the roots will be denoted with the same understanding: to be more explicit, if we write $X^{\alpha} X^{\beta} \alpha \cdot \beta$ we mean for example when $\alpha=1$ and $\beta=2 \rightarrow X^{1} X^{2} \alpha^{1} \cdot \alpha^{2}$. Notice in addition that the hermiticity condition gives e.g. $X_{k}^{-\alpha}=X_{k}^{* \alpha}$.

Now we make a specific choice of the background configuration, i.e. straight line trajectories for the three particles. This amounts to have $B_{k}$ in diagonal form with

$$
B_{k}^{\alpha}=\tilde{v}_{k}^{\alpha} \tau+\tilde{b}_{k}^{\alpha} \quad \alpha=1,2,3
$$

The free motion of the center of mass can be factored out and ignored imposing

$$
\sum_{\alpha=1}^{3} \tilde{v}_{k}^{\alpha}=0 \quad \sum_{\alpha=1}^{3} \tilde{b}_{k}^{\alpha}=0
$$

We introduce further simplifications restricting ourselves to the case of parallel velocities for all three particles, e.g. along the $x_{1}$ axis, and relative displacements transverse [7]

$$
\begin{array}{ll}
\tilde{v}_{1}^{\alpha} \neq 0 \quad, \quad \tilde{v}_{k}^{\alpha}=0 \quad \text { for } \quad k>1 \\
\sum_{k=1}^{9} \tilde{v}_{k}^{\alpha} \tilde{b}_{k}^{\alpha}=0 & \text { for } \quad \alpha=1,2,3
\end{array}
$$

Setting $\tilde{v}_{1}^{\alpha}=\tilde{v}_{\alpha}$ the background matrices become

$$
B_{1}=\left(\begin{array}{ccc}
\tilde{v}_{1} \tau & 0 & 0 \\
0 & \tilde{v}_{2} \tau & 0 \\
0 & 0 & \tilde{v}_{3} \tau
\end{array}\right) \quad, \quad B_{k}=\left(\begin{array}{ccc}
\tilde{b}_{k}^{1} & 0 & 0 \\
0 & \tilde{b}_{k}^{2} & 0 \\
0 & 0 & \tilde{b}_{k}^{3}
\end{array}\right) \quad \text { for } \quad k>1
$$

We define relative velocities

$$
v_{1}=\tilde{v}_{2}-\tilde{v}_{3} \quad \text { and } \quad \text { cyclic }
$$


and relative impact parameters

$$
b_{k}^{1}=\tilde{b}_{k}^{2}-\tilde{b}_{k}^{3} \quad \text { and } \quad \text { cyclic }
$$

In terms of these quantities, setting

$$
R_{k}^{\alpha}=\sum_{a=1,2} \alpha_{a} \operatorname{Tr}\left(H^{a} B_{k}\right)
$$

we obtain

$$
R_{k}^{\alpha}=\left\{\begin{array}{ccc}
v_{\alpha} \tau & \text { if } & k=1 \\
b_{k}^{\alpha} & \text { if } & k>1
\end{array}\right.
$$

Now we can go back to the action in (2.3) and perform the trace operation explicitly. Using the notation

$$
\begin{aligned}
\left(b^{\alpha}\right)^{2} & \equiv \sum_{k} b_{k}^{\alpha} b_{k}^{\alpha} \\
\left(R^{\alpha}\right)^{2} & \equiv \sum_{k} R_{k}^{\alpha} R_{k}^{\alpha} \equiv v_{\alpha}^{2} \tau^{2}+\left(b^{\alpha}\right)^{2}
\end{aligned}
$$

we obtain the following expressions:

terms involving the $X$ fields:

$$
\begin{aligned}
S_{X}=\int d & \left\{X_{k}^{a}\left(-\partial_{\tau}^{2}\right) X_{k}^{a}+2 X_{k}^{* \alpha}\left(-\partial_{\tau}^{2}+\left(R^{\alpha}\right)^{2}\right) X_{k}^{\alpha}-2 \sqrt{g}\left[\epsilon ^ { \alpha \beta \gamma } R _ { k } ^ { \gamma } \left(X_{k}^{\alpha} X_{j}^{\beta} X_{j}^{\gamma}\right.\right.\right. \\
& \left.\left.+X_{k}^{* \alpha} X_{j}^{* \beta} X_{j}^{* \gamma}\right)-2 R_{k}^{\alpha} X_{j}^{\alpha} X_{j}^{* \alpha} X_{k}^{a} \alpha_{a}+R_{k}^{\alpha} X_{j}^{\alpha} X_{k}^{* \alpha} X_{j}^{a} \alpha_{a}+R_{k}^{\alpha} X_{k}^{\alpha} X_{j}^{* \alpha} X_{j}^{a} \alpha_{a}\right] \\
-g & -2 X_{j}^{\alpha} X_{j}^{* \alpha}\left(X_{k}^{a} \alpha_{a}\right)^{2}-2 X_{k}^{a} \beta_{a} X_{j}^{\beta} X_{k}^{\alpha} X_{j}^{\gamma} \epsilon^{\alpha \beta \gamma}+2 X_{k}^{a} \beta_{a} X_{j}^{b} \beta_{b} X_{j}^{\beta} X_{k}^{* \beta} \\
- & 2 X_{k}^{a} \beta_{a} X_{j}^{* \beta} X_{k}^{* \alpha} X_{j}^{* \gamma} \epsilon^{\alpha \beta \gamma}-X_{k}^{\alpha} X_{k}^{* \eta} X_{j}^{\beta} X_{j}^{* \rho} \epsilon^{\alpha \beta \gamma} \epsilon^{\eta \rho \gamma} \\
+ & \left.\left.X_{k}^{\alpha} X_{j}^{* \alpha} X_{k}^{\beta} X_{j}^{* \beta}(\alpha \cdot \beta)-X_{k}^{\alpha} X_{j}^{* \alpha} X_{j}^{\beta} X_{k}^{* \beta}(\alpha \cdot \beta)\right]\right\}
\end{aligned}
$$

terms containing the gauge field $A$ :

$$
\begin{aligned}
& S_{A}=\int d\left\{A^{a}\left(-\partial_{\tau}^{2}\right) A^{a}+2 A^{* \alpha}\left(-\partial_{\tau}^{2}+\left(R^{\alpha}\right)^{2}\right) A^{\alpha}+4 i \partial_{\tau} R_{1}^{\alpha}\left(A^{* \alpha} X_{1}^{\alpha}-X_{1}^{* \alpha} A^{\alpha}\right)\right. \\
&-2 i \sqrt{g}\left[A^{a} \alpha_{a} X_{k}^{\alpha} \partial_{\tau} X_{k}^{* \alpha}-A^{a} \alpha_{a} \partial_{\tau} X_{k}^{\alpha} X_{k}^{* \alpha}+A^{\alpha} \alpha_{a} \partial_{\tau} X_{k}^{a} X_{k}^{* \alpha}-A^{\alpha} \alpha_{a} X_{k}^{a} \partial_{\tau} X_{k}^{* \alpha}\right. \\
&+\left.A^{* \alpha} \alpha_{a} \partial_{\tau} X_{k}^{\alpha} X_{k}^{a}-A^{* \alpha} \alpha_{a} \partial_{\tau} X_{k}^{a} X_{k}^{\alpha}+\epsilon^{\alpha \beta \gamma} A^{\alpha} X_{k}^{\beta} \partial_{\tau} X_{k}^{\gamma}-\epsilon^{\alpha \beta \gamma} A^{* \alpha} X_{k}^{* \beta} \partial_{\tau} X_{k}^{* \gamma}\right] \\
&-2 \sqrt{g}\left[R_{k}^{\alpha} A^{b} \alpha_{b}\left(X_{k}^{* \alpha} A^{\alpha}+X_{k}^{\alpha} A^{* \alpha}\right)-2 R_{k}^{\alpha} A^{\alpha} A^{* \alpha} X_{k}^{b} \alpha_{b}\right. \\
&\left.+\epsilon^{\alpha \beta \gamma} R_{k}^{\gamma}\left(X_{k}^{\alpha} A^{\beta} A^{\gamma}+X_{k}^{* \alpha} A^{* \beta} A^{* \gamma}\right)\right]
\end{aligned}
$$




$$
\begin{aligned}
-g[ & -2 A^{\beta} A^{* \beta}\left(X_{k}^{a} \beta_{a}\right)^{2}-2 \epsilon^{\alpha \beta \gamma}\left(X_{k}^{a} \beta_{a}\right)\left(X_{k}^{\alpha} A^{\beta} A^{\gamma}+X_{k}^{* \alpha} A^{* \beta} A^{* \gamma}\right) \\
& +2\left(X_{k}^{a} \beta_{a}\right)\left(A^{b} \beta_{b}\right)\left(A^{\beta} X_{k}^{* \beta}+A^{* \beta} X_{k}^{\beta}\right)-2 X_{k}^{* \beta} X_{k}^{\beta}\left(A^{a} \beta_{a}\right)^{2} \\
& +2 \epsilon^{\alpha \beta \gamma}\left(A^{b} \alpha_{b}\right)\left(X_{k}^{\alpha} X_{k}^{\gamma} A^{\beta}+X_{k}^{* \alpha} X_{k}^{* \gamma} A^{* \beta}\right)-2 \epsilon^{\alpha \beta \gamma} \epsilon^{\eta \rho \gamma} X_{k}^{\alpha} X_{k}^{* \eta} A^{\beta} A^{* \rho} \\
& \left.\left.+(\alpha \cdot \beta)\left(X_{k}^{\alpha} X_{k}^{\beta} A^{* \alpha} A^{* \beta}+X_{k}^{* \alpha} X_{k}^{* \beta} A^{\alpha} A^{\beta}-2 X_{k}^{\alpha} X_{k}^{* \beta} A^{* \alpha} A^{\beta}\right)\right]\right\}
\end{aligned}
$$

terms containing the fermions:

$$
\begin{aligned}
S_{\theta}= & \int \mathrm{d} \tau\left\{\theta^{a T}\left(\partial_{\tau}\right) \theta^{a}+2 \theta^{* \alpha T}\left(\partial_{\tau}-\gamma^{i} R_{i}^{\alpha}\right) \theta^{\alpha}\right. \\
& -2 i \sqrt{g}\left[\theta^{a T} \alpha_{a}\left(\theta^{* \alpha} A^{\alpha}-\theta^{\alpha} A^{* \alpha}\right)+\frac{1}{2} \epsilon^{\alpha \beta \gamma}\left(\theta^{* \alpha T} \theta^{* \beta} A^{* \gamma}-\theta^{\alpha T} \theta^{\beta} A^{\gamma}\right)\right. \\
& \left.+\theta^{* \alpha T} \theta^{\alpha} A^{a} \alpha_{a}\right]-2 \sqrt{g}\left[\theta^{a T} \alpha_{a} \gamma^{i}\left(X_{i}^{\alpha} \theta^{* \alpha}-X_{i}^{* \alpha} \theta^{\alpha}\right)+\theta^{* \alpha T} \gamma^{i} \theta^{\alpha} X_{i}^{a} \alpha_{a}\right. \\
& \left.+\frac{1}{2} \epsilon^{\alpha \beta \gamma}\left(\theta^{* \alpha T} \gamma^{i} \theta^{* \beta} X_{i}^{* \gamma}-\theta^{\alpha T} \gamma^{i} \theta^{\beta} X_{i}^{\gamma}\right]\right\}
\end{aligned}
$$

terms involving the ghosts:

$$
\begin{aligned}
S_{G}= & \int \mathrm{d} \tau\left\{2 \tilde{G}^{\alpha}\left(-\partial_{\tau}^{2}+\left(R^{\alpha}\right)^{2}\right) G^{\alpha}+2 \tilde{G}^{* \alpha}\left(-\partial_{\tau}^{2}+\left(R^{\alpha}\right)^{2}\right) G^{* \alpha}\right. \\
& +2 \tilde{G}^{a}\left(-\partial_{\tau}^{2}\right) G^{a}+2 \sqrt{g}\left[-R_{k}^{\alpha} \alpha_{b}\left(\tilde{G}^{\alpha} X_{k}^{\alpha}+\tilde{G}^{* \alpha} X_{k}^{* \alpha}\right) G^{b}\right. \\
& \left.+R_{k}^{\alpha} \alpha_{b} X_{k}^{b}\left(\tilde{G}^{\alpha} G^{\alpha}+\tilde{G}^{* \alpha} G^{* \alpha}\right)+\epsilon^{\alpha \beta \gamma} R_{k}^{\gamma}\left(\tilde{G}^{\gamma} G^{* \alpha} X_{k}^{* \beta}+\tilde{G}^{* \gamma} G^{\alpha} X_{k}^{\beta}\right)\right] \\
& +2 i \sqrt{g}\left[\partial_{\tau} \tilde{G}^{a} \alpha_{a}\left(G^{\alpha} A^{* \alpha}-G^{* \alpha} A^{\alpha}\right)+\left(\partial_{\tau} \tilde{G}^{\alpha} A^{\alpha}-\partial_{\tau} \tilde{G}^{* \alpha} A^{* \alpha}\right) G^{a} \alpha_{a}\right. \\
& \left.\left.-\left(\partial_{\tau} \tilde{G}^{\alpha} G^{\alpha}-\partial_{\tau} \tilde{G}^{* \alpha} G^{* \alpha}\right) A^{a} \alpha_{a}+\epsilon^{\alpha \beta \gamma}\left(\partial_{\tau} \tilde{G}^{* \gamma} G^{\alpha} A^{\beta}-\partial_{\tau} \tilde{G}^{\gamma} G^{* \alpha} A^{* \beta}\right)\right]\right\}
\end{aligned}
$$

From the quadratic part of the action one can easily read the mass matrix ( diagonalizing further (2.14) and (2.15) ) and obtain the following particle content in the spectrum:

- 8 complex bosons with mass $\left(R^{\alpha}\right)^{2}$ where $\alpha=1,2,3$;

- 1 complex boson with mass $\left(R^{\alpha}\right)^{2} \pm 2 v_{\alpha}$ where $\alpha=1,2,3$;

- 20 real, massless bosons;

- 2 complex ghost fields with mass $\left(R^{\alpha}\right)^{2}$ where $\alpha=1,2,3$;

- 2 real, massless ghosts; 
- 1 complex spinor with mass $\gamma^{i} R_{i}^{\alpha}$ where $\alpha=1,2,3$;

- 2 Majorana, massless spinors.

Correspondingly we obtain the propagators of the various fields 国. The bosonic ones are given by

$$
\begin{aligned}
<X_{i}^{a}(\tau) X_{j}^{b}\left(\tau^{\prime}\right)> & =\frac{1}{2} \delta^{a b} \delta_{i j} \Delta_{0}\left(\tau, \tau^{\prime}\right) \\
<A^{a}(\tau) A^{b}\left(\tau^{\prime}\right)> & =\frac{1}{2} \delta^{a b} \Delta_{0}\left(\tau, \tau^{\prime}\right) \\
<X_{i}^{* \alpha}(\tau) X_{j}^{\beta}\left(\tau^{\prime}\right)> & =\frac{1}{2} \delta^{\alpha \beta} \delta_{i j} \int_{0}^{\infty} \mathrm{d} s \Delta^{\alpha}\left(\tau, \tau^{\prime}, s\right) \quad i, j \neq 1 \\
<A^{* \alpha}(\tau) A^{\beta}\left(\tau^{\prime}\right)> & =<X_{1}^{* \alpha}(\tau) X_{1}^{\beta}\left(\tau^{\prime}\right)>=\frac{1}{2} \delta^{\alpha \beta} \int_{0}^{\infty} \mathrm{d} s \operatorname{Cosh}\left(2 v_{\alpha} s\right) \Delta^{\alpha}\left(\tau, \tau^{\prime}, s\right) \\
<X_{1}^{* \alpha}(\tau) A^{\beta}\left(\tau^{\prime}\right)> & =-<A^{* \alpha}(\tau) X_{1}^{\beta}\left(\tau^{\prime}\right)>=-i \frac{1}{2} \delta^{\alpha \beta} \int_{0}^{\infty} \mathrm{d} s \operatorname{Sinh}\left(2 v_{\alpha} s\right) \Delta^{\alpha}\left(\tau, \tau^{\prime}, s\right)
\end{aligned}
$$

while for the ghosts and the fermions we find

$$
\begin{aligned}
<\tilde{G}^{a}(\tau) G^{b}\left(\tau^{\prime}\right)> & =\frac{1}{2} \delta^{a b} \Delta_{0}\left(\tau, \tau^{\prime}\right) \\
<\tilde{G}^{\alpha}(\tau) G^{\beta}\left(\tau^{\prime}\right)> & =\frac{1}{2} \delta^{\alpha \beta} \int_{0}^{\infty} \mathrm{d} s \Delta^{\alpha}\left(\tau, \tau^{\prime}, s\right) \\
<\theta^{a T}(\tau) \theta^{b}\left(\tau^{\prime}\right)> & =\frac{1}{2} \delta^{a b} \partial_{\tau} \Delta_{0}\left(\tau, \tau^{\prime}\right) \\
<\theta^{* \alpha T}(\tau) \theta^{\beta}\left(\tau^{\prime}\right)> & =\frac{1}{2} \delta^{\alpha \beta} \Delta_{\mathbf{F}}^{\alpha}\left(\tau, \tau^{\prime}\right)
\end{aligned}
$$

We have defined

$$
\begin{aligned}
\Delta_{0}\left(\tau, \tau^{\prime}\right) & =\theta\left(\tau-\tau^{\prime}\right)\left(\tau^{\prime}-\tau\right) \\
\Delta_{\mathbf{F}}^{\alpha}\left(\tau, \tau^{\prime}\right) & \equiv \int_{0}^{\infty} \mathrm{d} s\left(-\mathbb{I} \frac{v_{\alpha} t}{\operatorname{Sinh}\left(v_{\alpha} s\right)}+\gamma^{1} \frac{v_{\alpha} T}{\operatorname{Cosh}\left(v_{\alpha} s\right)}\right.
\end{aligned}
$$




$$
\left.+\not{ }^{\alpha} \operatorname{Cosh}\left(v_{\alpha} s\right)+\not \phi^{\alpha} \gamma^{1} \operatorname{Sinh}\left(v_{\alpha} s\right)\right) \Delta^{\alpha}\left(\tau, \tau^{\prime}, s\right)
$$

where

$$
\Delta^{\alpha}\left(\tau, \tau^{\prime}, s\right)=e^{-\left(b^{\alpha}\right)^{2} s} \sqrt{\frac{v_{\alpha}}{2 \pi \operatorname{Sinh}\left(2 s v_{\alpha}\right)}} e^{-v_{\alpha} T^{2} \operatorname{Tanh}\left(s v_{\alpha}\right)-v_{\alpha} t^{2} \operatorname{Coth}\left(s v_{\alpha}\right)}
$$

We have introduced also new time variables

$$
T=\frac{1}{2}\left(\tau+\tau^{\prime}\right) \quad t=\frac{1}{2}\left(\tau-\tau^{\prime}\right)
$$

Once the propagators are known the one-loop contribution to the effective action is easily computed [1]

$$
\Gamma^{(1)}=-\frac{15}{16} \sum_{\alpha=1}^{3} \int d T \frac{\left(v_{\alpha}\right)^{4}}{\left[\left(b^{\alpha}\right)^{2}+T^{2}\left(v_{\alpha}\right)^{2}\right]^{\frac{7}{2}}}
$$

In the next section we present the two-loop calculation.

\section{The two-loop effective action}

The two-loop contributions are evaluated considering two types of diagrams involving one four-point vertex, the figure-eight graphs, and two three-point vertices, the sunsettype graphs, respectively. Given our setup, we can distinguish between the ones that lead to genuine three-body exchanges (one-particle irreducible diagrams for the threegraviton scattering in supergravity) and two-body recoil effects (one-particle reducible graphs in supergravity) in a very simple manner. We need collect terms that depend on two independent relative velocities on one side, and terms that depend only on one relative velocity on the other side.

Thus from the figure-eight diagrams we have a three-body exchange whenever the two propagators come in with different masses, while a recoil term is produced when the two masses are equal. The presence of a massless propagator would lead to a vanishing contribution for this tadpole kind of diagrams.

From the sunset-type graphs we have direct three-body contributions when the three propagators have three distinct masses (three different relative velocities out of which two are independent). Two-body forces are present when two masses are equal, being the 
third one equal to zero as required by momentum conservation. Having decomposed the fields as in (2.4) makes it easy to identify the massless particles which are given simply by the diagonal degrees of freedom.

Now we present our results. First we collect all the contributions to the three-body forces: we follow as much as possible the approach in ref. [6] so that a direct comparison can be made in a straightforward manner. Complete agreement with their findings is shown in the next subsection. Then we concentrate on the two-loop contributions that depend only on one relative velocity $v_{\alpha}$. Their complete evaluation is conceptually simple and algebraically manageable within our approach. We isolate the leading order term and prove its consistency with the result from two-graviton scattering obtained in [5]. This shows without any further ambiguity that we are dealing with recoil effects.

\subsection{Three-body contributions}

As emphasized above we need compute all the two-loop terms which depend on two distinct relative velocities, and this implies that only propagators associated to off-diagonal degrees of freedom (see (2.4) ) will enter this part of the calculation. We have to focus on four-point vertices for the figure-eight diagrams and three point interactions for the sunset diagrams: they are explicitly given in (2.14), (2.15), (2.16) and (2.17). The corresponding propagators can be read from $(2.18),(2.19)$ and $(2.20),(2.21)$. In order to keep the notation compact we write $\Delta^{\alpha}(s) \equiv \Delta^{\alpha}\left(\tau, \tau^{\prime}, s\right)$. The full result is given by

$$
\begin{aligned}
\Gamma=- & \sum_{\alpha \neq \beta \neq \gamma}\left[\int d T d s_{2} d s_{3} P_{0}\left(\Delta^{\beta}\left(s_{2}\right) \Delta^{\gamma}\left(s_{3}\right)\right)_{\mid t=0}\right. \\
& +\int d T d t d s_{1} d s_{2} d s_{3} P_{1}\left(\partial_{\tau} \partial_{\tau^{\prime}} \Delta^{\alpha}\left(s_{1}\right)\right) \Delta^{\beta}\left(s_{2}\right) \Delta^{\gamma}\left(s_{3}\right) \\
& +\int d T d t d s_{1} d s_{2} d s_{3} P_{2} \tau^{\prime}\left(\partial_{\tau} \Delta^{\alpha}\left(s_{1}\right)\right) \Delta^{\beta}\left(s_{2}\right) \Delta^{\gamma}\left(s_{3}\right) \\
& +\int d T d t d s_{1} d s_{2} d s_{3} P_{3} v_{\alpha}^{2} \tau \tau^{\prime} \Delta^{\alpha}\left(s_{1}\right) \Delta^{\beta}\left(s_{2}\right) \Delta^{\gamma}\left(s_{3}\right) \\
& +\int d T d t d s_{1} d s_{2} d s_{3} P_{4} v_{\alpha} v_{\beta} \tau \tau^{\prime} \Delta^{\alpha}\left(s_{1}\right) \Delta^{\beta}\left(s_{2}\right) \Delta^{\gamma}\left(s_{3}\right) \\
& +\int d T d t d s_{1} d s_{2} d s_{3} P_{5}\left(b^{\alpha}\right)^{2} \Delta^{\alpha}\left(s_{1}\right) \Delta^{\beta}\left(s_{2}\right) \Delta^{\gamma}\left(s_{3}\right) \\
& \left.+\int d T d t d s_{1} d s_{2} d s_{3} P_{6} b^{\alpha} b^{\beta} \Delta^{\alpha}\left(s_{1}\right) \Delta^{\beta}\left(s_{2}\right) \Delta^{\gamma}\left(s_{3}\right)\right]
\end{aligned}
$$

where the complete expressions of the various $P$ 's are given in Appendix B. 
At this point we perform manipulations similar to the ones introduced in ref. [6]: this will lead to an easy comparison of our results and theirs. Since

$$
b^{\alpha}+b^{\beta}+b^{\gamma}=0 \quad \quad v_{\alpha}+v_{\beta}+v_{\gamma}=0 \quad \alpha \neq \beta \neq \gamma
$$

we have

$$
\sum_{\alpha \neq \beta \neq \gamma} b^{\alpha} b^{\beta} f(\alpha, \beta, \gamma)=\sum_{\alpha \neq \beta \neq \gamma} \frac{1}{2}\left(b^{\alpha}\right)^{2}[f(\gamma, \beta, \alpha)-f(\alpha, \beta, \gamma)-f(\alpha, \gamma, \beta)]
$$

The identity in (3.3) allows to combine $P_{6}$ and $P_{5}$ : we rename the resulting expression $P_{5}^{\text {new }}$. In the same way, using again (3.3) we combine $P_{4}$ and $P_{3}$ and rename their sum $P_{3}^{\text {new }}$. Moreover as in [6] we can write

$$
\begin{gathered}
\int d T d s_{2} d s_{3} P\left(\Delta^{\beta}\left(s_{2}\right) \Delta^{\gamma}\left(s_{3}\right)\right)_{\mid t=0}=\int d T d t d s_{2} d s_{3} P \delta(t) \Delta^{\beta}\left(s_{2}\right) \Delta^{\gamma}\left(s_{3}\right) \\
=-\int d T d t d s_{1} d s_{2} d s_{3} P\left(\partial_{s_{1}} \Delta^{\alpha}\left(s_{1}\right)\right) \Delta^{\beta}\left(s_{2}\right) \Delta^{\gamma}\left(s_{3}\right)
\end{gathered}
$$

and use the following relation

$$
\begin{aligned}
\left(b^{\alpha}\right)^{2} \Delta^{\alpha}\left(s_{1}\right)=- & \partial_{s_{1}} \Delta^{\alpha}\left(s_{1}\right)+\left(-v_{\alpha}^{2} T^{2}-v_{\alpha}^{2} t^{2}+v_{\alpha}^{2} T^{2} \frac{\operatorname{Sinh}\left(s_{1} v_{\alpha}\right)^{2}}{\operatorname{Cosh}\left(s_{1} v_{\alpha}\right)^{2}}\right. \\
& \left.+v_{\alpha}^{2} t^{2} \frac{\operatorname{Cosh}\left(s_{1} v_{\alpha}\right)^{2}}{\operatorname{Sinh}\left(s_{1} v_{\alpha}\right)^{2}}-\frac{1}{2} v_{\alpha} \frac{\operatorname{Sinh}\left(s_{1} v_{\alpha}\right)}{\operatorname{Cosh}\left(s_{1} v_{\alpha}\right)}-\frac{1}{2} v_{\alpha} \frac{\operatorname{Cosh}\left(s_{1} v_{\alpha}\right)}{\operatorname{Sinh}\left(s_{1} v_{\alpha}\right)}\right) \Delta^{\alpha}\left(s_{1}\right)
\end{aligned}
$$

It is rather straightforward to show that, with the help of (3.3), (3.4) and (3.5), the answer in (3.1) can be rearranged as a sum of two contributions

$$
\Gamma=\Gamma_{V}+\Gamma_{Y}
$$

Here

$$
\begin{aligned}
\Gamma_{V}= & -\sum_{\beta \neq \gamma} \int d T d s_{2} d s_{3}\left(P_{5}^{\text {new }}{ }_{\mid s_{1}=0}+P_{0}\right)\left(\Delta^{\beta}\left(s_{2}\right) \Delta^{\gamma}\left(s_{3}\right)\right)_{\mid t=0} \\
= & -\sum_{\beta \neq \gamma} \int d T d s_{2} d s_{3} 128 \operatorname{Sinh}^{3}\left(\frac{s_{2} v_{\beta}}{2}\right) \operatorname{Sinh}^{3}\left(\frac{s_{3} v_{\gamma}}{2}\right) \times \\
& \left(2 \operatorname{Cosh}\left(\frac{s_{2} v_{\beta}}{2}\right) \operatorname{Cosh}\left(\frac{s_{3} v_{\gamma}}{2}\right)-\operatorname{Sinh}\left(\frac{s_{2} v_{\beta}}{2}\right) \operatorname{Sinh}\left(\frac{s_{3} v_{\gamma}}{2}\right)\right) \Delta^{\beta}\left(s_{2}\right) \Delta^{\gamma}\left(s_{3}\right)
\end{aligned}
$$


This result exactly reproduces the corresponding term obtained in ref. [6].

The second term in (3.6) is given by

$$
\begin{aligned}
& \Gamma_{Y}=-\sum_{\alpha \neq \beta \neq \gamma} \int d T d t d s_{1} d s_{2} d s_{3}\left(\frac{v_{\alpha}}{\operatorname{Sinh}\left(2 s_{1} v_{\alpha}\right)}+v_{\alpha}^{2} T^{2} \operatorname{Tanh}^{2}\left(s_{1} v_{\alpha}\right)-v_{\alpha}^{2} t^{2} \operatorname{Coth}^{2}\left(s_{1} v_{\alpha}\right)\right) \times \\
& P_{1} \Delta^{\alpha}\left(s_{1}\right) \Delta^{\beta}\left(s_{2}\right) \Delta^{\gamma}\left(s_{3}\right) \\
& +\int d T d t d s_{1} d s_{2} d s_{3}\left(-v_{\alpha} T^{2} \operatorname{Tanh}\left(s_{1} v_{\alpha}\right)+v_{\alpha} t^{2} \operatorname{Coth}\left(s_{1} v_{\alpha}\right)\right) \times \\
& P_{2} \Delta^{\alpha}\left(s_{1}\right) \Delta^{\beta}\left(s_{2}\right) \Delta^{\gamma}\left(s_{3}\right) \\
& +\int d T d t d s_{1} d s_{2} d s_{3} P_{3}^{n e w} v_{\alpha}^{2} \tau \tau^{\prime} \Delta^{\alpha}\left(s_{1}\right) \Delta^{\beta}\left(s_{2}\right) \Delta^{\gamma}\left(s_{3}\right) \\
& +\int d T d t d s_{1} d s_{2} d s_{3}\left(\partial_{s_{1}} P_{5}^{n e w}-\left(v_{\alpha}^{2} T^{2}+v_{\alpha}^{2} t^{2}\right) P_{5}^{n e w}\right) \Delta^{\alpha}\left(s_{1}\right) \Delta^{\beta}\left(s_{2}\right) \Delta^{\gamma}\left(s_{3}\right) \\
& +\int d T d t d s_{1} d s_{2} d s_{3}\left(v_{\alpha}^{2} T^{2} \frac{\operatorname{Sinh}\left(s_{1} v_{\alpha}\right)^{2}}{\operatorname{Cosh}\left(s_{1} v_{\alpha}\right)^{2}}+v_{\alpha}^{2} t^{2} \frac{\operatorname{Cosh}\left(s_{1} v_{\alpha}\right)^{2}}{\operatorname{Sinh}\left(s_{1} v_{\alpha}\right)^{2}}\right) \times \\
& P_{5}^{\text {new }} \Delta^{\alpha}\left(s_{1}\right) \Delta^{\beta}\left(s_{2}\right) \Delta^{\gamma}\left(s_{3}\right) \\
& +\int d T d t d s_{1} d s_{2} d s_{3}\left(-\frac{1}{2} v_{\alpha} \frac{\operatorname{Sinh}\left(s_{1} v_{\alpha}\right)}{\operatorname{Cosh}\left(s_{1} v_{\alpha}\right)}-\frac{1}{2} v_{\alpha} \frac{\operatorname{Cosh}\left(s_{1} v_{\alpha}\right)}{\operatorname{Sinh}\left(s_{1} v_{\alpha}\right)}\right) \times \\
& P_{5}^{\text {new }} \Delta^{\alpha}\left(s_{1}\right) \Delta^{\beta}\left(s_{2}\right) \Delta^{\gamma}\left(s_{3}\right)
\end{aligned}
$$

Further simplifications are introduced using the identity

$$
\int_{-\infty}^{+\infty} d r r^{2} e^{-Q r^{2}}=\int_{-\infty}^{+\infty} d r \frac{1}{2 Q} e^{-Q r^{2}}
$$

Indeed, recalling the expression of $\Delta^{\alpha}$ in $(2.21)$, the above identity allows to perform the following substitutions in the integrals in $T$ and $t$ :

$$
\begin{aligned}
T^{2} \Delta^{\alpha}\left(s_{1}\right) \Delta^{\beta}\left(s_{2}\right) \Delta^{\gamma}\left(s_{3}\right) & \rightarrow \frac{\Delta^{\alpha}\left(s_{1}\right) \Delta^{\beta}\left(s_{2}\right) \Delta^{\gamma}\left(s_{3}\right)}{2\left(v_{\alpha} \operatorname{Tanh}\left(v_{\alpha} s_{1}\right)+v_{\beta} \operatorname{Tanh}\left(v_{\beta} s_{2}\right)+v_{\gamma} \operatorname{Tanh}\left(v_{\gamma} s_{3}\right)\right)} \\
t^{2} \Delta^{\alpha}\left(s_{1}\right) \Delta^{\beta}\left(s_{2}\right) \Delta^{\gamma}\left(s_{3}\right) & \rightarrow \frac{\Delta^{\alpha}\left(s_{1}\right) \Delta^{\beta}\left(s_{2}\right) \Delta^{\gamma}\left(s_{3}\right)}{2\left(v_{\alpha} \operatorname{Coth}\left(v_{\alpha} s_{1}\right)+v_{\beta} \operatorname{Coth}\left(v_{\beta} s_{2}\right)+v_{\gamma} \operatorname{Coth}\left(v_{\gamma} s_{3}\right)\right)}
\end{aligned}
$$


Finally in (3.8) we can expand the various coefficients in front of the common factor $\Delta^{\alpha}\left(s_{1}\right) \Delta^{\beta}\left(s_{2}\right) \Delta^{\gamma}\left(s_{3}\right)$ in powers of $v$ and find the first non vanishing contribution at order $v^{8}$

$$
\begin{gathered}
\Gamma_{Y}=-\sum_{\alpha \neq \beta \neq \gamma} \int d T d t d s_{1} d s_{2} d s_{3} \frac{1}{18}\left(s_{1} v_{\alpha}-s_{2} v_{\beta}\right)^{2}\left(s_{1} v_{\alpha}-s_{3} v_{\gamma}\right)^{2}\left(s_{2} v_{\beta}-s_{3} v_{\gamma}\right)^{2} \\
\left(s_{1} v_{\alpha}^{2}+s_{2} v_{\beta}^{2}+s_{3} v_{\gamma}^{2}\right) \Delta^{\alpha}\left(s_{1}\right) \Delta^{\beta}\left(s_{2}\right) \Delta^{\gamma}\left(s_{3}\right)
\end{gathered}
$$

We note that in ref. [6] the corresponding leading contribution is at order $v^{6}$. With our choice of the background it is easy to check that the order $v^{6}$ term vanishes identically since all the relative velocities are parallel to each other. The order $v^{8}$ contribution is contained in the complete answer given in [6], but algebraically too difficult to be reconstructed. In our case it can be evaluated rather easily since it is the first non vanishing contribution.

Now we turn to the calculation of the recoil effects.

\subsection{Two-body recoil contributions}

We consider here all the diagrams not computed in the previous section, i.e. figure-eight graphs with the two propagators carrying the same mass, and sunset-type graphs with two propagators of equal masses and the third one massless. Looking at the spectrum of the various particles it is clear that these contributions depend on a single relative velocity $v_{\alpha}$ and are thus candidates to represent supegravity two-body recoil effects. In this section we determine them exactly and show that the above interpretation is indeed confirmed.

The complete amplitude can be written (cfr. (3.1)):

$$
\begin{aligned}
\Gamma_{\text {recoil }}=-\sum_{\alpha} & {\left[\int d T d s_{1} d s_{2} Q_{0}\left(\Delta^{\alpha}\left(s_{2}\right) \Delta^{\alpha}\left(s_{3}\right)\right)_{\mid t=0}\right.} \\
+ & +\int d T d t d s_{1} d s_{2} Q_{1} \Delta_{0}\left(\partial_{\tau} \partial_{\tau^{\prime}} \Delta^{\alpha}\left(s_{2}\right)\right) \Delta^{\alpha}\left(s_{1}\right) \\
& +\int d T d t d s_{1} d s_{2} Q_{2} \Delta_{0}\left(\partial_{\tau} \Delta^{\alpha}\left(s_{1}\right)\right)\left(\partial_{\tau^{\prime}} \Delta^{\alpha}\left(s_{2}\right)\right) \\
& +\int d T d t d s_{1} d s_{2} Q_{3} \Delta_{0}\left(v_{\alpha} \tau^{\prime} \partial_{\tau} \Delta^{\alpha}\left(s_{2}\right)\right) \Delta^{\alpha}\left(s_{2}\right) \\
& +\int d T d t d s_{1} d s_{2} Q_{4} v_{\alpha}^{2} \tau \tau^{\prime} \Delta_{0} \Delta^{\alpha}\left(s_{1}\right) \Delta^{\alpha}\left(s_{2}\right) \\
& +\int d T d t d s_{1} d s_{2} Q_{5}\left(b^{\alpha}\right)^{2} \Delta_{0} \Delta^{\alpha}\left(s_{1}\right) \Delta^{\alpha}\left(s_{2}\right)
\end{aligned}
$$




$$
\begin{aligned}
& +\int d T d t d s_{1} d s_{2} Q_{6} v_{\alpha}^{2} T^{2} \Delta_{0} \Delta^{\alpha}\left(s_{1}\right) \Delta^{\alpha}\left(s_{2}\right) \\
& +\int d T d t d s_{1} d s_{2} Q_{7} v_{\alpha}^{2} t^{2} \Delta_{0} \Delta^{\alpha}\left(s_{1}\right) \Delta^{\alpha}\left(s_{2}\right) \\
& \left.+\int d T d t d s_{1} d s_{2} Q_{8} \Delta_{0} \Delta^{\alpha}\left(s_{1}\right) \Delta^{\alpha}\left(s_{2}\right)\right]
\end{aligned}
$$

where the expressions of the $Q$ coefficients are given in Appendix C. If we compare (3.12) with the corresponding three-body exchange contributions in (3.1) we notice that in the present case each term contains only one $v_{\alpha}$. As before the figure-eight terms are the ones in which $Q_{0}$ appears; all the rest comes from sunset graphs with one massless propagator, $\Delta_{0}$.

As we have done for the three-body calculation we can simplify the form of the result. We use the following relation

$$
\begin{aligned}
& \int d T d s_{1} d s_{2} Q_{0}\left(\Delta^{\alpha}\left(s_{1}\right) \Delta^{\alpha}\left(s_{2}\right)\right)_{\mid t=0}=\int d T d t d s_{1} d s_{2} Q_{0} \delta(t) \Delta^{\alpha}\left(s_{1}\right) \Delta^{\alpha}\left(s_{2}\right) \\
& =\int d T d t d s_{1} d s_{1} Q_{0}\left(\partial_{\tau^{\prime}} \partial_{\tau} \Delta_{0}\right) \Delta^{\alpha}\left(s_{1}\right) \Delta^{\alpha}\left(s_{2}\right) \\
& =\int d T d t d s_{1} d s_{1} Q_{0} \Delta_{0}\left(\partial_{\tau^{\prime}} \partial_{\tau} \Delta^{\alpha}\left(s_{1}\right) \Delta^{\alpha}\left(s_{2}\right)+2 \partial_{\tau^{\prime}} \Delta^{\alpha}\left(s_{1}\right) \partial_{\tau} \Delta^{\alpha}\left(s_{2}\right)\right. \\
& \left.\quad+\Delta^{\alpha}\left(s_{1}\right) \partial_{\tau^{\prime}} \partial_{\tau} \Delta^{\alpha}\left(s_{2}\right)\right)
\end{aligned}
$$

Given the expressions of the propagators in (2.21), we are able to perform the derivatives in (3.12) explicitly. In addition, in complete analogy with what we have done in (3.9) and in (3.10), we can show that, since we are integrating over $T$ and $t$ the following substitutions are allowed

$$
\begin{aligned}
T^{2} \Delta_{0} \Delta^{\alpha}\left(s_{1}\right) \Delta^{\alpha}\left(s_{2}\right) & \longrightarrow \frac{1}{2 v_{\alpha}\left(\operatorname{Tanh}\left(v_{\alpha} s_{1}\right)+\operatorname{Tanh}\left(v_{\alpha} s_{2}\right)\right)} \Delta_{0} \Delta^{\alpha}\left(s_{1}\right) \Delta^{\alpha}\left(s_{2}\right) \\
t^{2} \Delta_{0} \Delta^{\alpha}\left(s_{1}\right) \Delta^{\alpha}\left(s_{2}\right) & \longrightarrow \frac{1}{v_{\alpha}\left(\operatorname{Coth}\left(v_{\alpha} s_{1}\right)+\operatorname{Coth}\left(v_{\alpha} s_{2}\right)\right)} \Delta_{0} \Delta^{\alpha}\left(s_{1}\right) \Delta^{\alpha}\left(s_{2}\right)
\end{aligned}
$$

The above substitutions lead to

$$
\Gamma_{\text {recoil }}=-\sum_{\alpha}\left[\int d T d t d s_{1} d s_{2}\left(\left(b^{\alpha}\right)^{2} S_{1}+S_{2}\right) \Delta_{0} \Delta^{\alpha}\left(s_{1}\right) \Delta^{\alpha}\left(s_{2}\right)\right]
$$

where $S_{1}$ and $S_{2}$ are given by

$$
S_{1}=40+2 \operatorname{Cosh}\left(2 s_{1} v_{\alpha}\right)+32 \operatorname{Cosh}\left(\left(s_{1}-s_{2}\right) v_{\alpha}\right)+2 \operatorname{Cosh}\left(2 s_{2} v_{\alpha}\right)
$$




$$
\begin{aligned}
& +96 \operatorname{Cosh}\left(\left(s_{1}+s_{2}\right) v_{\alpha}\right)+8 \operatorname{Cosh}\left(2\left(s_{1}+s_{2}\right) v_{\alpha}\right) \\
S_{2}=\frac{1}{4}[ & -99 v_{\alpha}+128 v_{\alpha} \operatorname{Cosh}\left(s_{1} v_{\alpha}\right)+37 v_{\alpha} \operatorname{Cosh}\left(2 s_{1} v_{\alpha}\right)-3 v_{\alpha} \operatorname{Cosh}\left(4 s_{1} v_{\alpha}\right) \\
& -384 v_{\alpha} \operatorname{Cosh}\left(\left(s_{1}-s_{2}\right) v_{\alpha}\right)-48 v_{\alpha} \operatorname{Cosh}\left(2\left(s_{1}-s_{2}\right) v_{\alpha}\right)+128 v_{\alpha} \operatorname{Cosh}\left(s_{2} v_{\alpha}\right) \\
& +37 v_{\alpha} \operatorname{Cosh}\left(2 s_{2} v_{\alpha}\right)-3 v_{\alpha} \operatorname{Cosh}\left(4 s_{2} v_{\alpha}\right)+384 v_{\alpha} \operatorname{Cosh}\left(\left(s_{1}+s_{2}\right) v_{\alpha}\right) \\
& +92 v_{\alpha} \operatorname{Cosh}\left(2\left(s_{1}+s_{2}\right) v_{\alpha}\right)+v_{\alpha} \operatorname{Cosh}\left(4\left(s_{1}+s_{2}\right) v_{\alpha}\right)-128 v_{\alpha} \operatorname{Cosh}\left(\left(2 s_{1}+s_{2}\right) v_{\alpha}\right) \\
& -7 v_{\alpha} \operatorname{Cosh}\left(2\left(2 s_{1}+s_{2}\right) v_{\alpha}\right)-128 v_{\alpha} \operatorname{Cosh}\left(\left(s_{1}+2 s_{2}\right) v_{\alpha}\right) \\
& \left.-7 v_{\alpha} \operatorname{Cosh}\left(2\left(s_{1}+2 s_{2}\right) v_{\alpha}\right)\right] \operatorname{Cosech}\left(s_{1} v_{\alpha}\right) \operatorname{Cosech}\left(s_{2} v_{\alpha}\right) \operatorname{Cosech}\left(\left(s_{1}+s_{2}\right) v_{\alpha}\right)
\end{aligned}
$$

At this stage we integrate on $t$ and $T$ explicitly and expand the result in power of $v_{\alpha}$ up to order $v_{\alpha}^{5}$. We find

$$
\Gamma_{\text {recoil }}=-\sum_{\alpha}\left[\int d s_{1} d s_{2} \frac{\left(\left(b^{\alpha}\right)^{2} V_{1}+V_{2}\right) e^{-\left(b^{\alpha}\right)^{2}\left(s_{1}+s_{2}\right)}}{2 \pi v_{\alpha} \sqrt{s_{1} s_{2}} \sqrt{s_{1}+s_{2}}}\right]
$$

where

$$
\begin{aligned}
V_{1}=( & 75600+22680 s_{1}^{2} v_{\alpha}^{2}+2520 s_{1} s_{2} v_{\alpha}^{2}+22680 s_{2}^{2} v_{\alpha}^{2}+630 s_{1}^{4} v_{\alpha}^{4}-2100 s_{1}^{3} s_{2} v_{\alpha}^{4} \\
& +10290 s_{1}^{2} s_{2}^{2} v_{\alpha}^{4}-2100 s_{1} s_{2}^{3} v_{\alpha}^{4}+630 s_{2}^{4} v_{\alpha}^{4}+139 s_{1}^{6} v_{\alpha}^{6}+761 s_{1}^{5} s_{2} v_{\alpha}^{6} \\
& \left.+1794 s_{1}^{4} s_{2}^{2} v_{\alpha}^{6}-947 s_{1}^{3} s_{2}^{3} v_{\alpha}^{6}+1794 s_{1}^{2} s_{2}^{4} v_{\alpha}^{6}+761 s_{1} s_{2}^{5} v_{\alpha}^{6}+139 s_{2}^{6} v_{\alpha}^{6}\right) \frac{s_{1} s_{2}}{840\left(s_{1}+s_{2}\right)} \\
V_{2}= & -75600 s_{1}^{2}+302400 s_{1} s_{2}-75600 s_{2}^{2}-17640 s_{1}^{4} v_{\alpha}^{2}-27720 s_{1}^{3} s_{2} v_{\alpha}^{2} \\
& -277200 s_{1}^{2} s_{2}^{2} v_{\alpha}^{2}-27720 s_{1} s_{2}^{3} v_{\alpha}^{2}-17640 s_{2}^{4} v_{\alpha}^{2}-6510 s_{1}^{6} v_{\alpha}^{4}+2940 s_{1}^{5} s_{2} v_{\alpha}^{4} \\
& +68040 s_{1}^{4} s_{2}^{2} v_{\alpha}^{4}+211680 s_{1}^{3} s_{2}^{3} v_{\alpha}^{4}+68040 s_{1}^{2} s_{2}^{4} v_{\alpha}^{4}+2940 s_{1} s_{2}^{5} v_{\alpha}^{4}-6510 s_{2}^{6} v_{\alpha}^{4} \\
& -97 s_{1}^{8} v_{\alpha}^{6}+2065 s_{1}^{7} s_{2} v_{\alpha}^{6}-7695 s_{1}^{6} s_{2}^{2} v_{\alpha}^{6}-51738 s_{1}^{5} s_{2}^{3} v_{\alpha}^{6}-103508 s_{1}^{4} s_{2}^{4} v_{\alpha}^{6} \\
& \left.-51738 s_{1}^{3} s_{2}^{5} v_{\alpha}^{6}-7695 s_{1}^{2} s_{2}^{6} v_{\alpha}^{6}+2065 s_{1} s_{2}^{7} v_{\alpha}^{6}-97 s_{2}^{8} v_{\alpha}^{6}\right) \frac{1}{3360\left(s_{1}+s_{2}\right)^{2}}
\end{aligned}
$$

Finally the integrations on $s_{1}$ and $s_{2}$ can be performed using the following relation

$$
\int d s_{1} d s_{2} \frac{s_{1}^{\ell} s_{2}^{m} e^{-\left(b^{\alpha}\right)^{2}\left(s_{1}+s_{2}\right)}}{\left(s_{1}+s_{2}\right)^{n} \sqrt{s_{1} s_{2}} \sqrt{s_{1}+s_{2}}}=\frac{1}{\left(b^{\alpha}\right)^{2 \ell+2 m-2 n+1}} \beta\left(\ell+\frac{1}{2}, m+\frac{1}{2}\right) \Gamma\left(\ell+m-n+\frac{1}{2}\right)
$$


Indeed with the help of (3.19) it is easy to show that

$$
\begin{aligned}
& \int d s_{1} d s_{2} \frac{\left(b^{\alpha}\right)^{2} V_{1} e^{-\left(b^{\alpha}\right)^{2}\left(s_{1}+s_{2}\right)}}{2 \pi v_{\alpha} \sqrt{s_{1} s_{2}} \sqrt{s_{1}+s_{2}}} \\
& =\frac{45 \pi\left(4194304\left(b^{\alpha}\right)^{12}+3047424\left(b^{\alpha}\right)^{8} v_{\alpha}^{2}+1241856\left(b^{\alpha}\right)^{4} v_{\alpha}^{4}+11390093 v_{\alpha}^{6}\right)}{67108864\left(b^{\alpha}\right)^{13} v_{\alpha}}
\end{aligned}
$$

and

$$
\begin{aligned}
& \int d s_{1} d s_{2} \frac{V_{2} e^{-\left(b^{\alpha}\right)^{2}\left(s_{1}+s_{2}\right)}}{2 \pi v_{\alpha} \sqrt{s_{1} s_{2}} \sqrt{s_{1}+s_{2}}} \\
& =-\frac{45 \pi\left(4194304\left(b^{\alpha}\right)^{12}+3047424\left(b^{\alpha}\right)^{8} v_{\alpha}^{2}+1241856\left(b^{\alpha}\right)^{4} v_{\alpha}^{4}+10207373 v_{\alpha}^{6}\right)}{67108864\left(b^{\alpha}\right)^{13} v_{\alpha}}
\end{aligned}
$$

Summing the two contributions in (3.20) and (3.21) we obtain

$$
\Gamma_{\text {recoil }}=-\sum_{\alpha} \frac{51975}{65536} \pi \frac{v_{\alpha}^{5}}{\left(b^{\alpha}\right)^{13}}
$$

We write $(3.22)$ in the form

$$
\Gamma_{\text {recoil }}=-\sum_{\alpha} \int d T \frac{225}{64} \frac{v_{\alpha}^{6}}{\left(\left(b^{\alpha}\right)^{2}+v_{\alpha}^{2} T^{2}\right)^{7}}
$$

The expression in (3.23) can be directly compared with the result in ref. [5] where the two graviton scattering was analyzed: we find that numbers perfectly match. The expression in (3.15) with $\Gamma_{V}$ in (3.7) and $\Gamma_{Y}$ in (3.8) determines the full two-loop contribution to the three $\mathrm{D}$-particle effective action.

\section{Conclusions}

In this paper we have presented a complete two-loop calculation in $M$-atrix theory. We have considered the gauge group $U(3)$, thus focusing on the description of three $D 0$ branes. In order to maintain the algebraic complexity of the computation under control, we have made a specific choice of the classical background, i.e. we have restricted our 
attention to the case of three $D$-particles whose relative velocities are parallel and orthogonal to the corresponding relative displacements. First we have confirmed the results obtained in [6], showing that the predictions from one-particle irreducible tree diagrams in supergravity are in agreement with two-loop contributions from $M$-atrix theory. Then we have considered those two-loop contributions which depend only on one relative velocity. The novelty of our result resides primarily in this part of our work. We have shown that this new type of terms gives a total sum which is exactly consistent with what is obtained from a two-loop calculation of two $D$-particle scattering [5]. Therefore we can safely conclude that we have determined those effects which amount to one-particle reducible tree level diagrams in supergravity. Once more one finds impressive evidence that elevendimensional supergravity compactified in a null direction is in direct correspondence with $M$-atrix theory results for finite $N$.

\section{Acknowledgments.}

This work was supported by the European Commission TMR program ERBFMRXCT96-0045, in which A. R., N. T. and D. Z. are associated to the University of Torino. 


\section{A Lie algebra for $S U(3)$}

We have chosen the following Cartan basis for the Lie algebra of $S U(3)$

$$
\begin{array}{ccc}
H^{1}= & \frac{1}{\sqrt{6}}\left(\begin{array}{ccc}
1 & 0 & 0 \\
0 & 1 & 0 \\
0 & 0 & -2
\end{array}\right) & H^{2}=\frac{1}{\sqrt{2}}\left(\begin{array}{ccc}
1 & 0 & 0 \\
0 & -1 & 0 \\
0 & 0 & 0
\end{array}\right) \\
E_{\alpha^{1}}=\left(\begin{array}{ccc}
0 & 0 & 0 \\
0 & 0 & 1 \\
0 & 0 & 0
\end{array}\right) & E_{-\alpha^{1}}=\left(\begin{array}{lll}
0 & 0 & 0 \\
0 & 0 & 0 \\
0 & 1 & 0
\end{array}\right) \\
E_{\alpha^{2}}=\left(\begin{array}{ccc}
0 & 0 & 0 \\
0 & 0 & 0 \\
1 & 0 & 0
\end{array}\right) & E_{-\alpha^{2}}=\left(\begin{array}{lll}
0 & 0 & 1 \\
0 & 0 & 0 \\
0 & 0 & 0
\end{array}\right) \\
E_{\alpha^{3}}=\left(\begin{array}{lll}
0 & 1 & 0 \\
0 & 0 & 0 \\
0 & 0 & 0
\end{array}\right) & E_{-\alpha^{3}}=\left(\begin{array}{lll}
0 & 0 & 0 \\
1 & 0 & 0 \\
0 & 0 & 0
\end{array}\right)
\end{array}
$$

with root vectors

$$
\alpha^{1}=\left(\sqrt{\frac{3}{2}},-\frac{1}{\sqrt{2}}\right), \quad \alpha^{2}=\left(-\sqrt{\frac{3}{2}},-\frac{1}{\sqrt{2}}\right), \quad \alpha^{3}=(0, \sqrt{2})
$$

normalized as

$$
\alpha \cdot \beta=\left\{\begin{array}{cll}
2 & \text { if } & \alpha=\beta \\
-1 & \text { if } & \alpha \neq \beta
\end{array} \quad \alpha^{1}+\alpha^{2}+\alpha^{3}=0\right.
$$

The generators satisfy the following commutation relations

$$
\begin{aligned}
{\left[H^{i}, E_{\alpha}\right] } & =\alpha_{i} E_{\alpha} & & {\left[H^{i}, E_{-\alpha}\right]=-\alpha_{i} E_{-\alpha} } \\
{\left[E_{\alpha^{1}}, E_{\alpha^{2}}\right] } & =E_{-\alpha^{3}} & {\left[E_{-\alpha^{1}}, E_{-\alpha^{2}}\right] } & =-E_{\alpha^{3}} \\
{\left[E_{\alpha^{2}}, E_{\alpha^{3}}\right] } & =E_{-\alpha^{1}} & & {\left[E_{-\alpha^{2}}, E_{-\alpha^{3}}\right]=-E_{\alpha^{1}} } \\
{\left[E_{\alpha^{3}}, E_{\alpha^{1}}\right] } & =E_{-\alpha^{2}} & & {\left[E_{-\alpha^{3}}, E_{-\alpha^{1}}\right]=-E_{\alpha^{2}} }
\end{aligned}
$$




$$
\begin{gathered}
{\left[E_{\alpha^{1}}, E_{-\alpha^{1}}\right]=\alpha^{1} \cdot H} \\
{\left[E_{\alpha^{2}}, E_{-\alpha^{2}}\right]=\alpha^{2} \cdot H} \\
{\left[E_{\alpha^{3}}, E_{-\alpha^{3}}\right]=\alpha^{3} \cdot H} \\
{\left[H^{i}, H^{j}\right]=0}
\end{gathered}
$$

\section{B Coefficients for three-body forces}

We list here the expressions of $P_{0}, \ldots, P_{6}$ which appear into the two-loop effective action for direct three-body exchanges.

$$
\begin{aligned}
& P_{0}=-\left(45+18 \operatorname{Sinh}\left(s_{3} v_{\gamma}\right)^{2}+18 \operatorname{Sinh}\left(s_{2} v_{\beta}\right)^{2}+4 \operatorname{Sinh}\left(s_{3} v_{\gamma}\right)^{2} \operatorname{Sinh}\left(s_{2} v_{\beta}\right)^{2}\right. \\
& \left.-12 \operatorname{Sinh}\left(s_{2} v_{\beta}\right) \operatorname{Sinh}\left(s_{3} v_{\gamma}\right) \operatorname{Cosh}\left(s_{2} v_{\beta}\right) \operatorname{Cosh}\left(s_{3} v_{\gamma}\right)\right) \\
& P_{1}=4\left(1+2 \operatorname{Sinh}\left(s_{2} v_{\beta}\right)^{2}\right)+4\left(1+2 \operatorname{Sinh}\left(s_{3} v_{\gamma}\right)^{2}\right) \\
& +\left(1+2 \operatorname{Sinh}\left(s_{1} v_{\alpha}\right)^{2}\right)\left(1+2 \operatorname{Sinh}\left(s_{2} v_{\beta}\right)^{2}\right)\left(1+2 \operatorname{Sinh}\left(s_{3} v_{\gamma}\right)^{2}\right) \\
& -4 \operatorname{Sinh}\left(s_{2} v_{\beta}\right) \operatorname{Sinh}\left(s_{3} v_{\gamma}\right) \operatorname{Cosh}\left(s_{2} v_{\beta}\right) \operatorname{Cosh}\left(s_{3} v_{\gamma}\right)\left(1+2 \operatorname{Sinh}\left(s_{1} v_{\alpha}\right)^{2}\right) \\
& -6 \operatorname{Sinh}\left(s_{2} v_{\beta}\right) \operatorname{Cosh}\left(s_{2} v_{\beta}\right) \operatorname{Sinh}\left(s_{3} v_{\gamma}\right) \operatorname{Cosh}\left(s_{3} v_{\gamma}\right)\left(1+2 \operatorname{Sinh}\left(s_{1} v_{\alpha}\right)^{2}\right) \\
& +2 \operatorname{Sinh}\left(s_{1} v_{\alpha}\right) \operatorname{Cosh}\left(s_{1} v_{\alpha}\right) \operatorname{Sinh}\left(s_{3} v_{\gamma}\right) \operatorname{Cosh}\left(s_{3} v_{\gamma}\right)\left(1+2 \operatorname{Sinh}\left(s_{2} v_{\beta}\right)^{2}\right) \\
& +2 \operatorname{Sinh}\left(s_{1} v_{\alpha}\right) \operatorname{Cosh}\left(s_{1} v_{\alpha}\right) \operatorname{Sinh}\left(s_{2} v_{\beta}\right) \operatorname{Cosh}\left(s_{2} v_{\beta}\right)\left(1+2 \operatorname{Sinh}\left(s_{3} v_{\gamma}\right)^{2}\right) \\
& +1 / 2\left(1+2 \operatorname{Sinh}\left(s_{1} v_{\alpha}\right)^{2}\right)\left(1+2 \operatorname{Sinh}\left(s_{2} v_{\beta}\right)^{2}\right)\left(1+2 \operatorname{Sinh}\left(s_{3} v_{\gamma}\right)^{2}\right) \\
& -7 / 2\left(1+2 \operatorname{Sinh}\left(s_{1} v_{\alpha}\right)^{2}\right)+7 / 2\left(1+2 \operatorname{Sinh}\left(s_{2} v_{\beta}\right)^{2}\right)+7 / 2\left(1+2 \operatorname{Sinh}\left(s_{3} v_{\gamma}\right)^{2}\right) \\
& +32\left(\operatorname{Cosh}\left(s_{1} v_{\alpha}\right) \operatorname{Cosh}\left(s_{2} v_{\beta}\right)+\operatorname{Sinh}\left(s_{1} v_{\alpha}\right) \operatorname{Sinh}\left(s_{2} v_{\beta}\right)\right) \\
& +32\left(\operatorname{Cosh}\left(s_{1} v_{\alpha}\right) \operatorname{Cosh}\left(s_{3} v_{\gamma}\right)+\operatorname{Sinh}\left(s_{1} v_{\alpha}\right) \operatorname{Sinh}\left(s_{3} v_{\gamma}\right)\right) \\
& -32\left(\operatorname{Cosh}\left(s_{2} v_{\beta}\right) \operatorname{Cosh}\left(s_{3} v_{\gamma}\right)+\operatorname{Sinh}\left(s_{2} v_{\beta}\right) \operatorname{Sinh}\left(s_{3} v_{\gamma}\right)\right) \\
& P_{2}=v_{\alpha}\left(-16 \operatorname{Sinh}\left(s_{1} v_{\alpha}\right) \operatorname{Sinh}\left(s_{2} v_{\beta}\right) \operatorname{Sinh}\left(s_{3} v_{\gamma}\right) \operatorname{Cosh}\left(s_{1} v_{\alpha}\right) \operatorname{Cosh}\left(s_{2} v_{\beta}\right) \operatorname{Cosh}\left(s_{3} v_{\gamma}\right)\right. \\
& +4 \operatorname{Sinh}\left(s_{1} v_{\alpha}\right) \operatorname{Cosh}\left(s_{1} v_{\alpha}\right)\left(1+2 \operatorname{Sinh}\left(s_{2} v_{\beta}\right)^{2}\right)\left(1+2 \operatorname{Sinh}\left(s_{3} v_{\gamma}\right)^{2}\right) \\
& \left.+16 \operatorname{Sinh}\left(s_{3} v_{\gamma}\right) \operatorname{Cosh}\left(s_{3} v_{\gamma}\right)+16 \operatorname{Sinh}\left(s_{2} v_{\beta}\right) \operatorname{Cosh}\left(s_{2} v_{\beta}\right)\right) \\
& +v_{\beta}\left(-4 \operatorname{Sinh}\left(s_{1} v_{\alpha}\right) \operatorname{Cosh}\left(s_{1} v_{\alpha}\right)\left(1+2 \operatorname{Sinh}\left(s_{2} v_{\beta}\right)^{2}\right)\left(1+2 \operatorname{Sinh}\left(s_{3} v_{\gamma}\right)^{2}\right)\right. \\
& -4 \operatorname{Sinh}\left(s_{2} v_{\beta}\right) \operatorname{Cosh}\left(s_{2} v_{\beta}\right)\left(1+2 \operatorname{Sinh}\left(s_{1} v_{\alpha}\right)^{2}\right)\left(1+2 \operatorname{Sinh}\left(s_{3} v_{\gamma}\right)^{2}\right) \\
& +4 \operatorname{Sinh}\left(s_{3} v_{\gamma}\right) \operatorname{Cosh}\left(s_{3} v_{\gamma}\right)\left(1+2 \operatorname{Sinh}\left(s_{2} v_{\beta}\right)^{2}\right)\left(1+2 \operatorname{Sinh}\left(s_{1} v_{\alpha}\right)^{2}\right)
\end{aligned}
$$




$$
\begin{aligned}
& +16 \operatorname{Sinh}\left(s_{1} v_{\alpha}\right) \operatorname{Sinh}\left(s_{2} v_{\beta}\right) \operatorname{Sinh}\left(s_{3} v_{\gamma}\right) \operatorname{Cosh}\left(s_{1} v_{\alpha}\right) \operatorname{Cosh}\left(s_{2} v_{\beta}\right) \operatorname{Cosh}\left(s_{3} v_{\gamma}\right) \\
& -32 \operatorname{Sinh}\left(s_{3} v_{\gamma}\right) \operatorname{Cosh}\left(s_{3} v_{\gamma}\right)+4 \operatorname{Sinh}\left(s_{3} v_{\gamma}\right) \operatorname{Cosh}\left(s_{3} v_{\gamma}\right) \\
& \left.-128\left(\operatorname{Cosh}\left(s_{1} v_{\alpha}\right) \operatorname{Sinh}\left(s_{2} v_{\beta}\right)+\operatorname{Cosh}\left(s_{2} v_{\beta}\right) \operatorname{Sinh}\left(s_{1} v_{\alpha}\right)\right)\right) \\
& P_{3}=\left(1+2 \operatorname{Sinh}\left(s_{1} v_{\alpha}\right)^{2}\right)\left(1+2 \operatorname{Sinh}\left(s_{2} v_{\beta}\right)^{2}\right)\left(1+2 \operatorname{Sinh}\left(s_{3} v_{\gamma}\right)^{2}\right) \\
& -4\left(1+2 \operatorname{Sinh}\left(s_{1} v_{\alpha}\right)^{2}\right) \operatorname{Sinh}\left(s_{2} v_{\beta}\right) \operatorname{Sinh}\left(s_{3} v_{\gamma}\right) \operatorname{Cosh}\left(s_{2} v_{\beta}\right) \operatorname{Cosh}\left(s_{3} v_{\gamma}\right) \\
& +4\left(1+2 \operatorname{Sinh}\left(s_{2} v_{\beta}\right)^{2}\right)+4\left(1+2 \operatorname{Sinh}\left(s_{3} v_{\gamma}\right)^{2}\right) \\
& P_{4}=4\left(1+2 \operatorname{Sinh}\left(s_{1} v_{\alpha}\right)^{2}\right) \operatorname{Sinh}\left(s_{2} v_{\beta}\right) \operatorname{Sinh}\left(s_{3} v_{\gamma}\right) \operatorname{Cosh}\left(s_{2} v_{\beta}\right) \operatorname{Cosh}\left(s_{3} v_{\gamma}\right) \\
& +4\left(1+2 \operatorname{Sinh}\left(s_{2} v_{\beta}\right)^{2}\right) \operatorname{Sinh}\left(s_{1} v_{\alpha}\right) \operatorname{Sinh}\left(s_{3} v_{\gamma}\right) \operatorname{Cosh}\left(s_{1} v_{\alpha}\right) \operatorname{Cosh}\left(s_{3} v_{\gamma}\right) \\
& -4\left(1+2 \operatorname{Sinh}\left(s_{3} v_{\gamma}\right)^{2}\right) \operatorname{Sinh}\left(s_{2} v_{\beta}\right) \operatorname{Sinh}\left(s_{1} v_{\alpha}\right) \operatorname{Cosh}\left(s_{2} v_{\beta}\right) \operatorname{Cosh}\left(s_{1} v_{\alpha}\right) \\
& -\left(1+2 \operatorname{Sinh}\left(s_{1} v_{\alpha}\right)^{2}\right)\left(1+2 \operatorname{Sinh}\left(s_{2} v_{\beta}\right)^{2}\right)\left(1+2 \operatorname{Sinh}\left(s_{3} v_{\gamma}\right)^{2}\right) \\
& -8\left(1+2 \operatorname{Sinh}\left(s_{3} v_{\gamma}\right)^{2}\right)+\left(1+2 \operatorname{Sinh}\left(s_{3} v_{\gamma}\right)^{2}\right) \\
& -64\left(\operatorname{Sinh}\left(s_{1} v_{\alpha}\right) \operatorname{Sinh}\left(s_{2} v_{\beta}\right)+\operatorname{Cosh}\left(s_{1} v_{\alpha}\right) \operatorname{Cosh}\left(s_{2} v_{\beta}\right)\right) \\
& P_{5}=7+\left(1+2 \operatorname{Sinh}\left(s_{1} v_{\alpha}\right)^{2}\right)\left(1+2 \operatorname{Sinh}\left(s_{2} v_{\beta}\right)^{2}\right) \\
& +\left(1+2 \operatorname{Sinh}\left(s_{1} v_{\alpha}\right)^{2}\right)\left(1+2 \operatorname{Sinh}\left(s_{3} v_{\gamma}\right)^{2}\right) \\
& -4 \operatorname{Sinh}\left(s_{2} v_{\beta}\right) \operatorname{Sinh}\left(s_{1} v_{\alpha}\right) \operatorname{Cosh}\left(s_{2} v_{\beta}\right) \operatorname{Cosh}\left(s_{1} v_{\alpha}\right) \\
& -4 \operatorname{Sinh}\left(s_{1} v_{\alpha}\right) \operatorname{Sinh}\left(s_{3} v_{\gamma}\right) \operatorname{Cosh}\left(s_{1} v_{\alpha}\right) \operatorname{Cosh}\left(s_{3} v_{\gamma}\right) \\
& P_{6}=-\left(1+2 \operatorname{Sinh}\left(s_{1} v_{\alpha}\right)^{2}\right)\left(1+2 \operatorname{Sinh}\left(s_{2} v_{\beta}\right)^{2}\right)-6 \\
& -\left(1+2 \operatorname{Sinh}\left(s_{1} v_{\alpha}\right)^{2}\right)\left(1+2 \operatorname{Sinh}\left(s_{2} v_{\beta}\right)^{2}\right) \\
& +8 \operatorname{Sinh}\left(s_{1} v_{\alpha}\right) \operatorname{Sinh}\left(s_{2} v_{\beta}\right) \operatorname{Cosh}\left(s_{1} v_{\alpha}\right) \operatorname{Cosh}\left(s_{2} v_{\beta}\right)+48 \operatorname{Sinh}\left(s_{1} v_{\alpha}\right) \operatorname{Sinh}\left(s_{2} v_{\beta}\right) \\
& -48 \operatorname{Cosh}\left(s_{1} v_{\alpha}\right) \operatorname{Cosh}\left(s_{2} v_{\beta}\right) \\
& -16\left(1+2 \operatorname{Sinh}\left(s_{3} v_{\gamma}\right)^{2}\right)\left(\operatorname{Sinh}\left(s_{1} v_{\alpha}\right) \operatorname{Sinh}\left(s_{2} v_{\beta}\right)+\operatorname{Cosh}\left(s_{1} v_{\alpha}\right) \operatorname{Cosh}\left(s_{2} v_{\beta}\right)\right) \\
& +32 \operatorname{Cosh}\left(s_{3} v_{\gamma}\right) \operatorname{Sinh}\left(s_{3} v_{\gamma}\right)\left(\operatorname{Sinh}\left(s_{1} v_{\alpha}\right) \operatorname{Cosh}\left(s_{2} v_{\beta}\right)+\operatorname{Cosh}\left(s_{1} v_{\alpha}\right) \operatorname{Sinh}\left(s_{2} v_{\beta}\right)\right)
\end{aligned}
$$




\section{Coefficients for two-body forces}

We list here the expressions of $Q_{0}, \ldots, Q_{8}$ which appear into the two-loop effective action for two-body recoil exchanges.

$$
\begin{aligned}
& Q_{0}=-90-36 \operatorname{Sinh}\left(s_{2} v_{\alpha}\right)^{2}-36 \operatorname{Sinh}\left(s_{1} v_{\alpha}\right)^{2}-8 \operatorname{Sinh}\left(s_{1} v_{\alpha}\right)^{2} \operatorname{Sinh}\left(s_{2} v_{\alpha}\right)^{2} \\
& -24 \operatorname{Sinh}\left(s_{1} v_{\alpha}\right) \operatorname{Sinh}\left(s_{2} v_{\alpha}\right) \operatorname{Cosh}\left(s_{1} v_{\alpha}\right) \operatorname{Cosh}\left(s_{2} v_{\alpha}\right) \\
& Q_{1}=16+12\left(1+2 \operatorname{Sinh}\left(s_{2} v_{\alpha}\right)^{2}\right)\left(1+2 \operatorname{Sinh}\left(s_{1} v_{\alpha}\right)^{2}\right) \\
& +32 \operatorname{Sinh}\left(s_{1} v_{\alpha}\right) \operatorname{Sinh}\left(s_{2} v_{\alpha}\right) \operatorname{Cosh}\left(s_{1} v_{\alpha}\right) \operatorname{Cosh}\left(s_{2} v_{\alpha}\right) \\
& +60\left(1+2 \operatorname{Sinh}\left(s_{1} v_{\alpha}\right)^{2}\right)+16\left(1+2 \operatorname{Sinh}\left(s_{2} v_{\alpha}\right)^{2}\right) \\
& Q_{2}=-14+6\left(1+2 \operatorname{Sinh}\left(s_{2} v_{\alpha}\right)^{2}\right)\left(1+2 \operatorname{Sinh}\left(s_{1} v_{\alpha}\right)^{2}\right) \\
& +40 \operatorname{Sinh}\left(s_{1} v_{\alpha}\right) \operatorname{Sinh}\left(s_{2} v_{\alpha}\right) \operatorname{Cosh}\left(s_{1} v_{\alpha}\right) \operatorname{Cosh}\left(s_{2} v_{\alpha}\right) \\
& +30\left(1+2 \operatorname{Sinh}\left(s_{1} v_{\alpha}\right)^{2}\right)+30\left(1+2 \operatorname{Sinh}\left(s_{2} v_{\alpha}\right)^{2}\right) \\
& Q_{3}=2\left[4 \operatorname{Sinh}\left(s_{1} v_{\alpha}\right) \operatorname{Cosh}\left(s_{1} v_{\alpha}\right)\left(1+2 \operatorname{Sinh}\left(s_{2} v_{\alpha}\right)^{2}\right)\right. \\
& +20 \operatorname{Sinh}\left(s_{2} v_{\alpha}\right) \operatorname{Cosh}\left(s_{2} v_{\alpha}\right)\left(1+2 \operatorname{Sinh}\left(s_{1} v_{\alpha}\right)^{2}\right) \\
& \left.-60 \operatorname{Sinh}\left(s_{1} v_{\alpha}\right) \operatorname{Cosh}\left(s_{1} v_{\alpha}\right)-28 \operatorname{Sinh}\left(s_{2} v_{\alpha}\right) \operatorname{Cosh}\left(s_{2} v_{\alpha}\right)\right] \\
& Q_{4}=30+6\left(1+2 \operatorname{Sinh}\left(s_{2} v_{\alpha}\right)^{2}\right)\left(1+2 \operatorname{Sinh}\left(s_{1} v_{\alpha}\right)^{2}\right) \\
& -8 \operatorname{Sinh}\left(s_{1} v_{\alpha}\right) \operatorname{Sinh}\left(s_{2} v_{\alpha}\right) \operatorname{Cosh}\left(s_{1} v_{\alpha}\right) \operatorname{Cosh}\left(s_{2} v_{\alpha}\right) \\
& +8\left(1+2 \operatorname{Sinh}\left(s_{1} v_{\alpha}\right)^{2}\right)+8\left(1+2 \operatorname{Sinh}\left(s_{2} v_{\alpha}\right)^{2}\right) \\
& Q_{5}=40+8\left(1+2 \operatorname{Sinh}\left(s_{2} v_{\alpha}\right)^{2}\right)\left(1+2 \operatorname{Sinh}\left(s_{1} v_{\alpha}\right)^{2}\right) \\
& +32 \operatorname{Sinh}\left(s_{1} v_{\alpha}\right) \operatorname{Sinh}\left(s_{2} v_{\alpha}\right) \operatorname{Cosh}\left(s_{1} v_{\alpha}\right) \operatorname{Cosh}\left(s_{2} v_{\alpha}\right) \\
& +2\left(1+2 \operatorname{Sinh}\left(s_{1} v_{\alpha}\right)^{2}\right)+2\left(1+2 \operatorname{Sinh}\left(s_{2} v_{\alpha}\right)\right) \\
& +128 \operatorname{Cosh}\left(s_{1} v_{\alpha}\right) \operatorname{Cosh}\left(s_{2} v_{\alpha}\right)+64 \operatorname{Sinh}\left(s_{1} v_{\alpha}\right) \operatorname{Sinh}\left(s_{2} v_{\alpha}\right) \\
& Q_{6}=\frac{128}{\operatorname{Cosh}\left(s_{1} v_{\alpha}\right) \operatorname{Cosh}\left(s_{2} v_{\alpha}\right)}+\frac{128 \operatorname{Sinh}\left(s_{2} v_{\alpha}\right)^{2}}{\operatorname{Cosh}\left(s_{1} v_{\alpha}\right)} \\
& +128 \frac{\operatorname{Sinh}\left(s_{1} v_{\alpha}\right) \operatorname{Sinh}\left(s_{2} v_{\alpha}\right) \operatorname{Cosh}\left(s_{1} v_{\alpha}\right)}{\operatorname{Cosh}\left(s_{2} v_{\alpha}\right)^{2}}
\end{aligned}
$$




$$
\begin{gathered}
Q_{7}=\frac{128}{\operatorname{Sinh}\left(s_{1} v_{\alpha}\right) \operatorname{Sinh}\left(s_{2} v_{\alpha}\right)}-\frac{256 \operatorname{Cosh}\left(s_{2} v_{\alpha}\right)}{\operatorname{Sinh}\left(s_{1} v_{\alpha}\right) \operatorname{Sinh}\left(s_{2} v_{\alpha}\right)}-\frac{256 \operatorname{Cosh}\left(s_{2} v_{\alpha}\right)}{\operatorname{Sinh}\left(s_{2} v_{\alpha}\right)^{2}} \\
Q_{8}=\frac{64 v_{\alpha}}{\operatorname{Sinh}\left(s_{2} v_{\alpha}\right)}+\frac{64 v_{\alpha}}{\operatorname{Sinh}\left(s_{1} v_{\alpha}\right)}-\frac{32 v_{\alpha}}{\operatorname{Cosh}\left(s_{2} v_{\alpha}\right)} \operatorname{Sinh}\left(s_{1} v_{\alpha}\right) \operatorname{Cosh}\left(s_{1} v_{\alpha}\right) \\
\quad-\frac{32 v_{\alpha}}{\operatorname{Cosh}\left(s_{1} v_{\alpha}\right)} \operatorname{Sinh}\left(s_{2} v_{\alpha}\right) \operatorname{Cosh}\left(s_{2} v_{\alpha}\right)
\end{gathered}
$$




\section{References}

[1] T. Banks, W. Fischler, S.H. Shenker, L. Susskind, Phys. Rev. D55 (1997) 5112, hep-th/9610043

[2] L. Susskind, "Another conjecture about M(atrix) Theory", hep-th/9704080

[3] N. Seiberg, Phys. Rev. Lett. 79 (1997) 3577, hep-th/9710009

A. Sen, Adv.Theor.Math.Phys. 2 (1998) 51, hep-th/9709220

[4] K. Becker, M. Becker, Nucl. Phys. B506 (1997) 48, hep-th/9705091

[5] K. Becker, M. Becker, J. Polchinski, A. Tseytlin, Phys. Rev. D56 (1997) 3174, hep-th/9706072

[6] Y. Okawa, T. Yoneya, Nucl. Phys. B538 (1999) 67, hep-th/9806108; Nucl. Phys. B541 (1999) 163, hep-th/9808188

[7] M. Fabbrichesi, G. Ferretti, R. Iengo, J.H.E.P. 06 (1998) 2, hep-th/9806018

[8] W. Taylor, M. Raamsdonk, Phys. Lett. B438 (1998) 248, hep-th/9806066

R. Echols, J. Grey, Phys.Lett. B449 (1999) 60 hep-th/9806109

J. McCarthy, L. Susskind, A. Wilkins, Phys. Lett. B437 (1998) 62, hep-th/9806136

[9] M. Dine, A. Rajaraman, Phys. Lett. 425 (1998) 77, hep-th/9710174

[10] M. Claudson, M. Halpern, Nucl. Phys. B250 (1985) 689

R. Flume, Ann. Phys. 164 (1985) 189

M. Baake, P. Reinecke, V. Rittenberg, J. Math. Phys. 26 (1985) 1070

[11] J. Polchinski, Phys. Rev. Lett. 75 (1995) 4724, hep-th/950017

E. Witten, Nucl. Phys. B460 (1996) 335, hep-th/9510135 